\title{
Wireless Power Transmission for Autonomous Sensors in Removable Vehicle Seats
}

\author{
Joan Albesa and Manel Gasulla \\ Department of Electronic Engineering, ISI Group \\ Universitat Politècnica de Catalunya (UPC) \\ Barcelona, Spain
}

\author{
Thomas Jäger and Leonhard M. Reindl \\ Department of Microsystems Engineering, IMTEK \\ Albert-Ludwigs-Universität Freiburg \\ Freiburg, Germany
}

\begin{abstract}
This work proposes the use of magnetic coupling for powering autonomous sensors in space-constrained applications, such as occupancy and belt detection in removable vehicle seats. The power demand of the autonomous sensor is considered between tens and hundreds of milliwatts. A theoretical analysis first highlights the critical parameters in order to achieve a large powering range and high efficiency. Series-resonant tanks are considered for both the primary and secondary networks. Because the intended application is space-constrained, small coils have to be used. In order to increase their quality factor, commercial ferrite-core coils are used. A class D power amplifier is proposed for the primary network. Experimental results show that a power of tens of milliwatts can be transferred to a $100 \Omega$ load placed at the secondary network up to a distance of $2 \mathrm{~cm}$, near seven times the radius of the coils $(3 \mathrm{~mm})$. The addition of a rectifier and a voltage regulator at the secondary network in order to properly power an autonomous sensor $(3 \mathrm{~V} @ 30 \mathrm{~mA})$ limits the powering range to $1 \mathrm{~cm}$. Overall power efficiencies around $45 \%$ and $20 \%$ are achieved respectively at distances of $5 \mathrm{~mm}$ and $1 \mathrm{~cm}$.
\end{abstract}

Index Terms - Wireless Power Transfer, Contactless Energy Transfer, Inductive Powering, Autonomous Sensors, Vehicles, Removable Seats.

\section{INTRODUCTION}

Remote or wireless power transmission via inductive coupling has been around for a long time. High-power transfer includes battery recharging of electrical vehicles [1] and a broad range of industrial applications [2] whereas low-power transfer includes RFID systems [3], biomedical implants [4], or portable consumer electronic products [5].

Transmission power distance is, in general, shorter than the diameter of the powering coils. Recently, though, the possibility of effectively powering at higher distances has been demonstrated. In particular, a power transfer of $60 \mathrm{~W}$ with $40 \%$ coil-to-coil (60 $\mathrm{cm}$ in diameter) efficiency over distances in excess of $2 \mathrm{~m}$ ( $c a .3$ times the diameter of the coils) was shown in [6]. The analysis is rather based on physical theory and more engineering focused approaches using circuit lumped circuits have appeared since then [7]. The same principle has also been explored for powering: multiple receivers from a single transmitter coil [8], biomedical implants [9], or even chips [10].

Vehicles can also benefit from inductive powering. In particular, some vans and minivans incorporate removable seats in order to flexibly arrange their internal space. Wiring that seats in order to incorporate, for instance, seat belt

Joan Albesa enjoys a grant from the Ministry of Economy and Knowledge of the Regional Government of Catalonia, Spain, and the European Social Funding on the FI program. He also enjoyed a Mobility grant from the Ministry of Education of Spain and an UPC-Enterprise grant from the car company SEAT and the Technical University of Catalonia (UPC). detectors, can become unpractical. So, in some vehicles a passive detection is performed via magnetic coupling. The addition of wireless power could allow the incorporation of new devices that require some amount of power, such as a seat occupancy sensor or a microcontroller that adds intelligence to the removable seat. In these applications, the available or acceptable space for the coils is rather limited.

This paper explores the feasibility of remotely powering, via magnetic coupling, autonomous sensors for occupancy and belt detection in removable vehicle seats. The primary and secondary networks may be placed respectively in the car floor and at the bottom of the removable seat. Thus, the application is space-constrained and, consequently, small-size coils have to be used. In addition, the power demand of the load is considered between tens and hundreds of milliwatts, according to commercial radio devices used in autonomous sensors. In another paper, we propose seat occupancy and belt detection via a passive inductive link [11].

Section II first presents a theoretical analysis of the power transferred to the load with a pair of magnetic coupled resonators. Powering range distance and power efficiency are also analyzed. Then, Section III presents the selected commercial coils. Quality factors are measured for different frequencies and exposure regulations are verified through simulations. Section IV presents the design of the primary and secondary networks. The primary network includes a class D power amplifier whereas the secondary network includes a rectifier stage and a voltage regulator in order to properly power the autonomous sensor. Section V shows the measured performance. Finally, Section VI concludes the work.

\section{THEORETICAL ANALYSIS}

Fig. 1 shows the equivalent circuit of a pair of magnetically coupled series resonators, being the left-hand and right-hand networks the primary and secondary, respectively. $V_{1}$ is a sinusoidal signal that models the output of the power amplifier that drives the primary network; $I_{1}$ and $I_{2}$ stand for the currents of the primary and secondary; $L_{1}$ and $L_{2}$ model the coils; $C_{1}$ and $C_{2}$ are the added capacitances to work at resonance; $R_{\mathrm{S}}$ models the output resistance of the power amplifier, $R_{\mathrm{L} 1}$ and $R_{\mathrm{L} 2}$ model the losses of the coils, and $R_{\mathrm{Load}}$ models the load; and finally $M$ models the mutual inductance between the coils, where

$$
M=k \sqrt{L_{1} L_{2}}
$$


being $k$ the coupling factor between the coils. We neglect the losses of the capacitors as they usually are much lower than that of inductors.

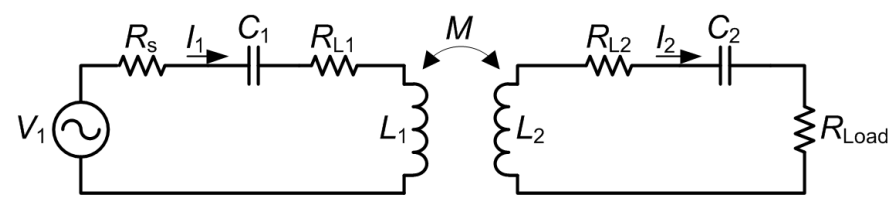

Figure 1. Equivalent circuit of a pair magnetically coupled series resonators.

The series-resonant primary network maximizes the injected current across $L_{1}$. For the secondary, a series-resonant tank was also chosen.

Assuming the same resonance frequency at the primary and secondary, that is

$\omega_{r}=\frac{1}{\sqrt{L_{1} C_{1}}}=\frac{1}{\sqrt{L_{2} C_{2}}}$

the power received at the load will be

$$
P_{\text {Load }}=\left(\frac{V_{1}}{R_{1}+R_{\text {eq }}}\right)^{2} R_{\text {eq }} \frac{R_{\text {Load }}}{R_{2}}
$$

where $V_{1}$ is assumed as the root-mean-square (rms) value of the voltage source, $R_{1}=R_{\mathrm{s}}+R_{\mathrm{L} 1}, R_{2}=R_{\mathrm{L} 2}+R_{\mathrm{Load}}$ and $R_{\mathrm{eq}}$ is the reflected resistance of the secondary onto the primary, which is given by

$R_{\mathrm{eq}}=\frac{(\omega M)^{2}}{R_{2}}$.

Eq. (3) can be rewritten as a function of quality factors

$P_{\text {Load }}=\frac{V_{1}^{2}}{R_{1}} \frac{k^{2} Q_{1} Q_{2}}{\left(1+k^{2} Q_{1} Q_{2}\right)^{2}} \frac{Q_{2}}{Q_{\text {Load }}}$

where

$Q_{1}=\frac{1}{R_{1}} \sqrt{\frac{L_{1}}{C_{1}}}$

$Q_{\text {Load }}=\frac{1}{R_{\text {Load }}} \sqrt{\frac{L_{2}}{C_{2}}}$.

$Q_{2}=\frac{1}{R_{2}} \sqrt{\frac{L_{2}}{C_{2}}}$

The overall power efficiency, defined as $P_{\text {Load }}$ divided by the generated power at the primary, is found as

$\eta_{\mathrm{T}}=\eta_{1} \eta_{2}$

where

$\eta_{1}=\frac{R_{\mathrm{eq}}}{R_{\mathrm{eq}}+R_{1}}=\frac{k^{2} Q_{1} Q_{2}}{1+k^{2} Q_{1} Q_{2}}$

$\eta_{2}=\frac{R_{\text {Load }}}{R_{2}}=\frac{Q_{2}}{Q_{\text {Load }}}$ $\eta_{1}$ accounts for the percentage of the generated power transferred to the secondary whereas $\eta_{2}$ accounts for the percentage of the received power at the secondary that is further transferred to the load. Both $\eta_{1}$ and $\eta_{2}$ must be high in order to have a high overall efficiency.

From (5) and (8) we can rewrite $P_{\text {Load }}$ as

$P_{\text {Load }}=\frac{V_{1}^{2}}{R_{1}} \eta_{1}\left(1-\eta_{1}\right) \eta_{2}$

The maximum of $P_{\text {Load }}$ can be found by solving $\partial P_{\text {Load }} / \partial k=0$, obtaining

$k_{\mathrm{c}}=\frac{1}{\sqrt{Q_{1} Q_{2}}}$.

The parameter $k_{\mathrm{c}}$ is referred as critical coupling [12]. Whenever $k=k_{\mathrm{c}}, \eta_{1}=0.5$, i.e. $R_{\mathrm{eq}}=R_{1}$, thus maximum power is transferred from the primary to the secondary circuit, being the load power

$P_{\text {Load,max }}=\frac{V_{1}^{2}}{4 R_{1}} \eta_{2}$.

The parameter $d_{\mathrm{c}}$ can be defined as the critical distance between the coils at which (10) holds. A lower $k_{\mathrm{c}}$ leads to a higher $d_{\mathrm{c}}$. By increasing $Q_{1}$ and $Q_{2}$ in (10), $k_{\mathrm{c}}$ is reduced and $d_{\mathrm{c}}$ is increased, thus increasing the powering distance range. For $k>k_{\mathrm{c}}$, i.e. $d<d_{\mathrm{c}}, P_{\mathrm{L}}$ decreases but $\eta_{1}$ monotonically increases towards the unity. The parameter $\eta_{2}$ does not depend on $k$ and thus remains constant over the distance.

\section{COILS, COUPLING FACTOR AND FIELD EXPOSURE}

For the intended application, small-size coils are sought. At the same time, in order to comply with the reference levels for general public exposure to time-varying electric and magnetic fields [13], and to reduce the power losses of the power amplifier, frequency resonance was limited to less than $150 \mathrm{kHz}$. So, in order to increase the quality factor of the coils with these constraints, the use of magnetic-core material was considered as an appropriate solution.

We selected commercial devices from Fastron (PIST model) with a value of $1 \mathrm{mH}$. These coils use ferrite as magnetic core material. Sizes can be shown in Fig. 2 (values are in millimeters). Published dc resistances are approximately $1.5 \Omega$.
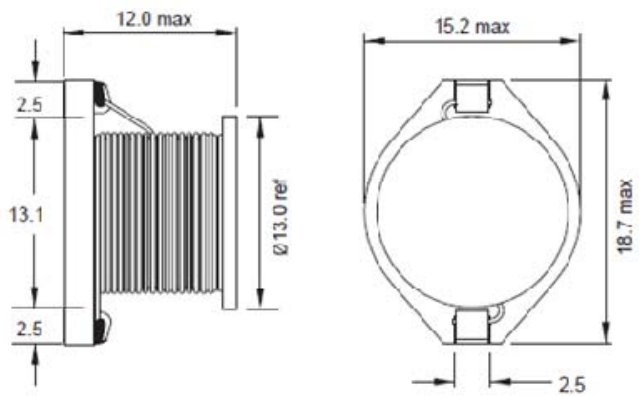

Figure 2. Dimensions of the selected $1 \mathrm{mH}$ coils (in millimeters). Source: http://www.fastrongroup.com/. 
In order to obtain the experimental quality factors of the coils at different frequencies, we used a series-resonant network and measured the resistance at the resonant frequency by using an HP4194A impedance analyzer. Appropriate values of capacitors were used in order to tune the resonant frequency. Fig. 3 shows both the measured resistances and the resulting quality factors. As can be seen, resistance values increased with frequency, which is due to the joint combination of skin and proximity effects and the losses of the ferrite [14]. Quality factor increased steeply at low frequencies achieving a maximum (ca. 40) around $40 \mathrm{kHz}$.

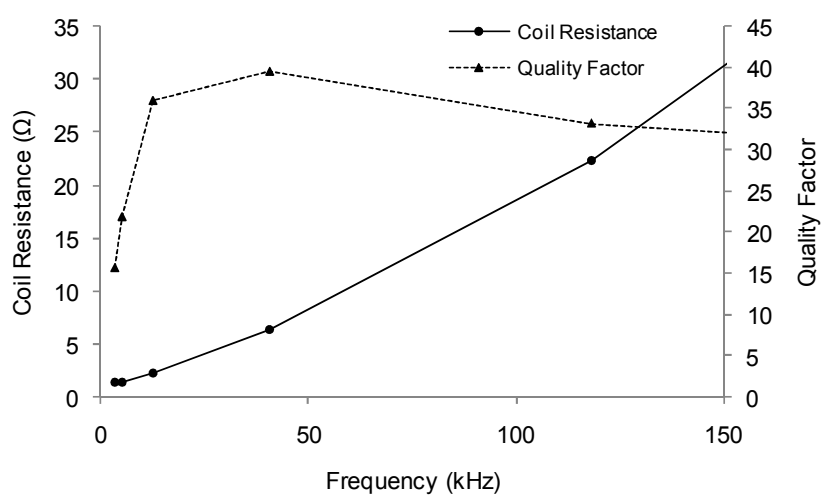

Figure 3. Values of resistance and quality factor for the selected commercial coil of $1 \mathrm{mH}$.

In order to estimate the coupling factor $(k)$ over the separation distance $(d)$ of the coils, we used the simulation program COMSOL. Fig. 4 shows an axisymetric model for the primary and secondary coils, and illustrates the parameter $d$. Sizes of the inductors were in accordance with those presented in Figure 2. The contour areas R1 to R6 were defined as ferrite whereas $\mathrm{C} 1$ and $\mathrm{C} 2$ (wire coil) were defined as copper. A relative permittivity $\left(\mu_{\mathrm{r}}\right)$ of 2000 was used for the ferrite. Spherical domain boundaries were used and set to zero magnetic insulation.

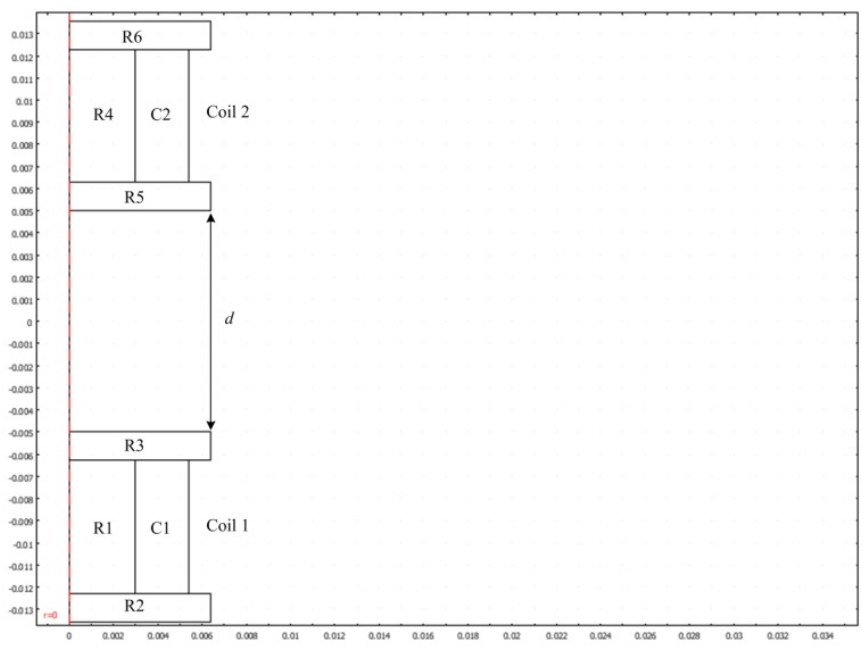

Figure 4. Modeling of the primary and secondary coils using COMSOL.
Fig. 5 shows the simulated results of $k$ over $d$. As can be seen, $k$ steeply increased for shorter distances.

Simulations were carried out in order to assess whether we complied with the reference levels for general public exposure to time-varying electric and magnetic fields [13]. An H-field strength lower than $5 \mathrm{~A} / \mathrm{m}$ (reference level for frequencies lower than $150 \mathrm{kHz}$ ) is achieved at distances higher than $0.5 \mathrm{~mm}$ from the coils, which is safe enough for the intended application. We used a suitable current density in accordance with the experimental results presented later in section $\mathrm{V}$.

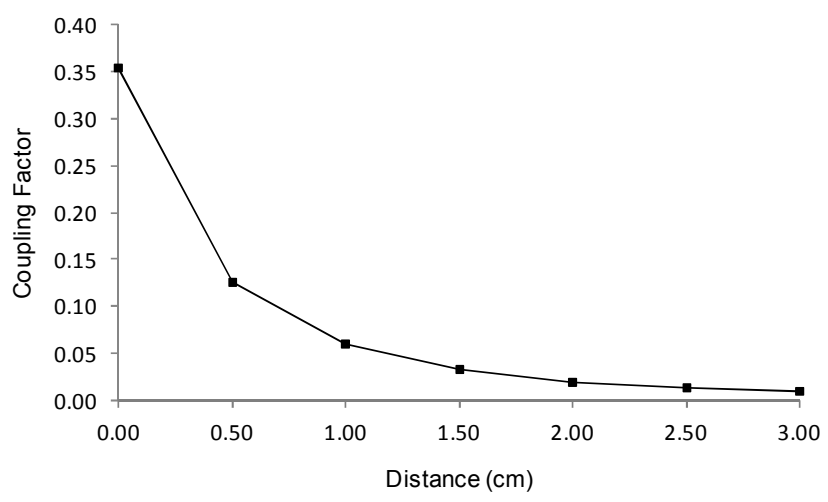

Figure 5. Evolution of $k$ over $d$.

\section{CIRCUIT DESIGN}

\section{A. Primary Network}

Fig. 6 shows the circuit schematic of the primary network. We used a class D power amplifier based on a low-cost commercial self-oscillating half-bridge driver (IR2153) and two external N-channel MOSFETs (BS108), M1 and M2. The driver, powered $\left(V_{\mathrm{cc}}\right)$ at $12 \mathrm{~V}$ dc (battery voltage in vehicles), alternatively activates the two MOSFETs, thus injecting a square wave signal into the series-resonant network. The oscillation frequency is selectable via an RC network $\left(R_{\mathrm{b}}, C_{\mathrm{b}}\right)$ up to $1 \mathrm{MHz}$. A potentiometer was used to fine tune the desired resonant frequency. Following the manufacturer guidelines, a bootstrap capacitor $\left(C_{\mathrm{c}}\right)$ was used to properly activate M1.

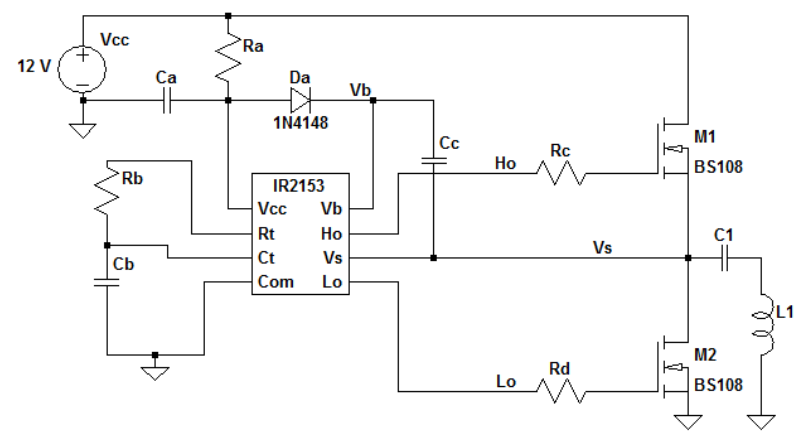

Figure 6. Circuit schematic of the primary network.

For moderate to high quality factors, only the first harmonic will generate a current through the network, being its amplitude of $7.64 \mathrm{~V}\left(2 V_{\mathrm{cc}} / \pi\right)$ and its rms value of $5.4 \mathrm{~V}$. 
The MOSFET manufacturer publishes an on resistance of $8 \Omega\left(@ V_{\mathrm{GS}}=2.8 \mathrm{~V}\right)$. In our case, we measured a lower value, around $4 \Omega$, due to the higher value of $V_{\mathrm{GS}}$. This value corresponds to $R_{\mathrm{s}}$ in Figure 1 .

\section{B. Secondary Network}

Fig. 7 shows the schematic circuit of the secondary network. As can be seen, in order to obtain a DC signal, a full bridge rectifier was jointly used with a stabilization capacitor $\left(C_{\mathrm{d}}\right)$. An ensuing voltage regulator (LP2980) was added to provide a voltage $\left(V_{\mathrm{L}}\right)$ of $3 \mathrm{~V}$ across the load $\left(R_{\mathrm{L}}\right)$. This voltage value is appropriate for low-power commercial transceivers, where current consumption is in the order of units to tens of milliamps. Here we consider a range of $3 \mathrm{~mA}$ to $30 \mathrm{~mA}$. This leads to an equivalent $R_{\mathrm{L}}$ of $100 \Omega$ to $1 \mathrm{k} \Omega$. A $10 \mathrm{~V}$ zener diode $D_{\mathrm{e}}$ was used for protecting the voltage regulator from overvoltages.

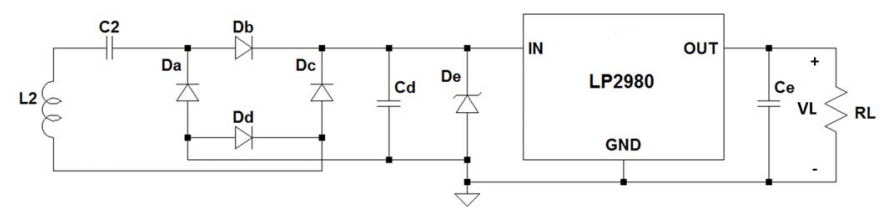

Figure 7. Circuit schematic of the secondary network.

\section{EXPERIMENTAL SETUP AND RESULTS}

Fig. 8 shows the mechanical setup fabricated to fix the distance between the primary and secondary networks, which were implemented in two separate PCB boards. The main support and the fixing screws were made of nylon. For the measurements, distance was adjusted manually from $0 \mathrm{~cm}$ to $3 \mathrm{~cm}$ in $0.5 \mathrm{~cm}$ steps. For the series-resonant networks, we used the commercial coils of $1 \mathrm{mH}$ presented in section III and capacitors $\left(C_{1}, C_{2}\right)$ of $1.8 \mathrm{nF}$. The frequency of the primary driver was adjusted to the experimental resonant frequency.

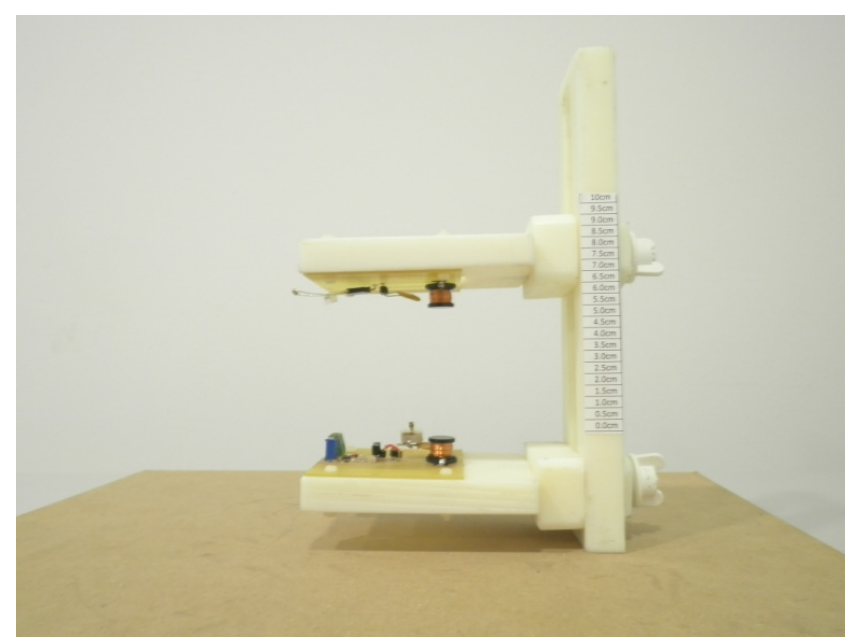

Figure 8. Mechanical setup used to fix the distance between the primary and the secondary networks.

First, only the resonant tank illustrated in Fig. 1 was used at the secondary network. Resistors of $100 \Omega$ and $1 \mathrm{k} \Omega$ were used for $R_{\text {Load. }}$. For each distance both $P_{\text {Load }}$ and $\eta_{\mathrm{T}}$ were estimated.
$P_{\text {Load }}$ was estimated by measuring the voltage drop across $R_{\text {Load }}$ with a floating oscilloscope. $\eta_{\mathrm{T}}$ was estimated by dividing $P_{\text {Load }}$ by the generated power from the $12 \mathrm{~V} \mathrm{dc}$ source of the primary network.

Fig. 9 shows $P_{\text {Load. }}$ As can be seen, the critical distance, $d_{\mathrm{c}}$, increased from $0.5 \mathrm{~cm}$ for $R_{\mathrm{Load}}=1 \mathrm{k} \Omega$ to $1 \mathrm{~cm}$ for $R_{\text {Load }}=100 \Omega$. This agrees with the theoretical predictions and simulations of section II and III. From (6), an increase of $R_{\mathrm{Load}}$ leads to a decrease of $Q_{2}$. Thus, from (10), $k_{\mathrm{c}}$ decreases leading to an increase of $d_{\mathrm{c}}$ (see Fig. 5). The value of $P_{\mathrm{L}, \max }$ for $R_{\text {Load }}=100 \Omega$ was $138 \mathrm{~mW}$, which nearly agrees with the predicted value of $134 \mathrm{~mW}$ obtained from (11). This predicted value was obtained considering $V_{1}=5.4 \mathrm{~V}, R_{\mathrm{s}}=4 \Omega$ (see section IV), and $R_{\mathrm{L} 1}=36 \Omega$. The value of $R_{\mathrm{L} 1}$ was estimated by measuring the voltage drop across a resistor momentarily added in series with the resonant tank. At a distance of $2 \mathrm{~cm}$, near seven times the internal radius of the coils $(3 \mathrm{~mm}), c a .55$ $\mathrm{mW}$ were transmitted to the load.

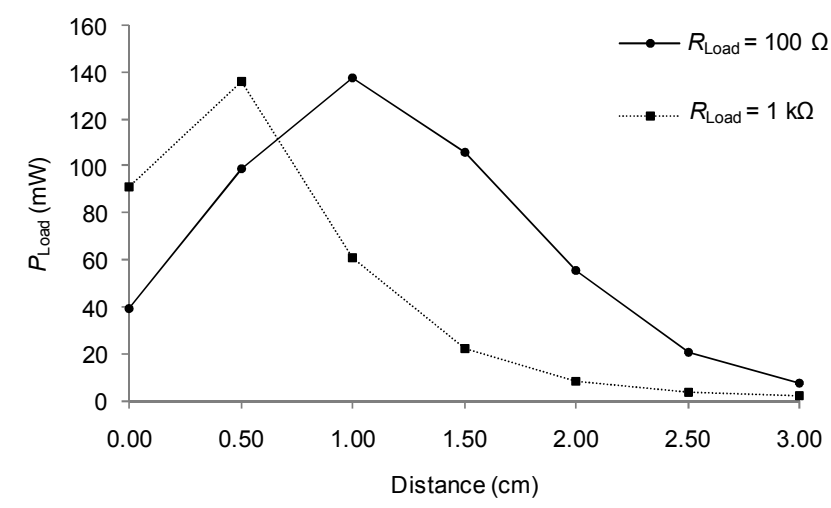

Figure 9. $P_{\text {Load }}$ for $L_{1}=L_{2}=1 \mathrm{mH}$ at a resonance frequency of $117 \mathrm{kHz}$.

Fig. 10 shows power efficiency. As can be seen, efficiency was higher for $R_{\text {Load }}=100 \Omega$ than for $R_{\text {Load }}=1 \mathrm{k} \Omega$ down to 0.5 $\mathrm{cm}$. Efficiencies for $R_{\mathrm{Load}}=100 \Omega$ at $0.5 \mathrm{~cm}$ and $1 \mathrm{~cm}$ were around $50 \%$ and $40 \%$, respectively. Conversely, for a distance of $0 \mathrm{~cm}$ efficiency decreased for $R_{\text {Load }}=100 \Omega$ because of the losses of the half-bridge driver of the primary network, $c a$. $15 \mathrm{~mW}$. This power loss was relatively less important for $R_{\text {Load }}=1 \mathrm{k} \Omega$ because the higher value of the load power at $0 \mathrm{~cm}$ (see Fig. 9), achieving an efficiency near $60 \%$.

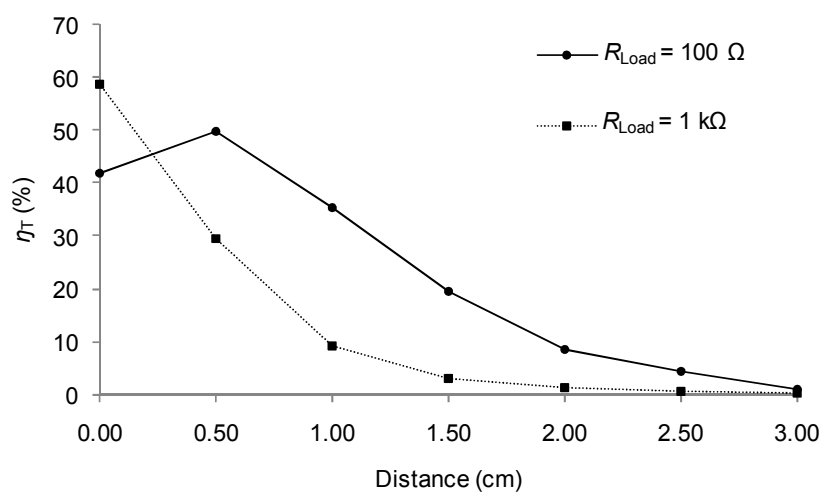

Figure 10. $\eta_{\mathrm{T}}$ for $L_{1}=L_{2}=1 \mathrm{mH}$ at a resonance frequency of $117 \mathrm{kHz}$ 
Then, the circuit of Fig. 7 was used at the secondary network with resistors of $100 \Omega, 1 \mathrm{k} \Omega$, and $1 \mathrm{M} \Omega$ for $R_{\mathrm{L}}$. Now, for each distance, $V_{\mathrm{L}}$ (Figure 11. ) was measured and power efficiency was again estimated (Fig. 12). For $R_{\text {Load }}=100 \Omega$, the desired voltage of $3 \mathrm{~V}$, and thus a load power of $90 \mathrm{~mW}$, was achieved for distances from $0.5 \mathrm{~cm}$ to $1 \mathrm{~cm}$. Corresponding efficiencies ranged from $\mathrm{ca}$. $45 \%$ to $20 \%$. For $R_{\text {Load }}=1 \mathrm{k} \Omega$, the desired voltage of $3 \mathrm{~V}$, and thus a load power of $9 \mathrm{~mW}$, was achieved for distances up to $1.5 \mathrm{~cm}$. Efficiencies were rather low in this case. Additionally, $R_{\text {Load }}=1 \mathrm{M} \Omega$ was considered, which emulates the case when the autonomous sensor demands a low current $(3 \mu \mathrm{A})$, i.e. is in a sleep mode. Here, a suitable voltage was also achieved for distances up to $1.5 \mathrm{~cm}$. Efficiency was now extremely low, which is logical considering the losses of the primary driver and the low power demanded by the load $(9 \mu \mathrm{W})$.

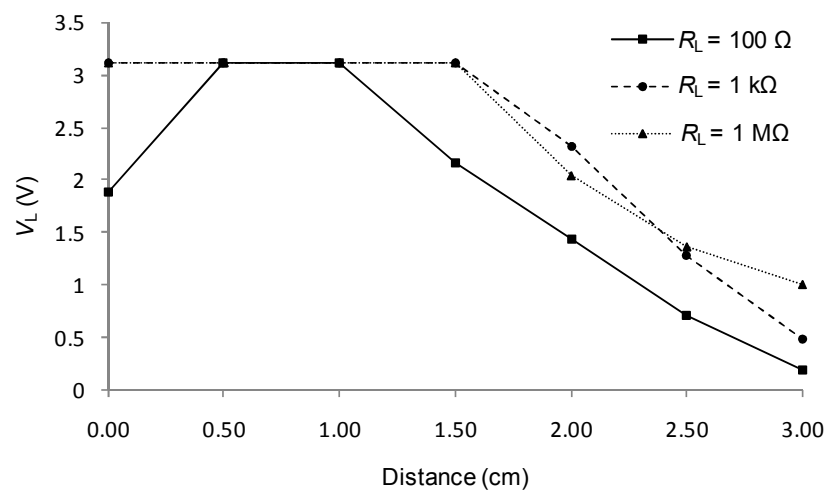

Figure 11. Load voltage $\left(V_{\mathrm{L}}\right)$ across $R_{\mathrm{L}}$ for the secodary network of Fig.7.

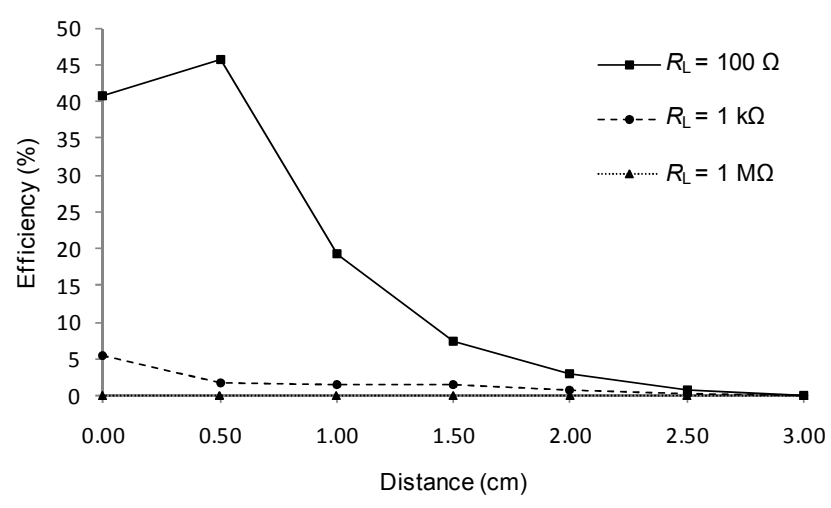

Figure 12. Overall efficiency for the secodary network of Fig. 7.

\section{CONCLUSIONS}

The principle of magnetic coupling resonance has been recently proposed to power portable devices. Here, we have used such a principle for exploring the possibility of powering autonomous sensors, e.g. seat belt and occupancy detectors, in removable vehicle seats. First, a theoretical analysis highlights the need of using high quality coils in order to achieve a large powering range and high efficiency. Additionally, the application is space-constrained. Thus, commercial ferrite-core coils of small-size have been used. The quality factor of the coils has been measured and simulations have demonstrated that the magnetic field is below the reference levels for general public exposure at distances higher than $5 \mathrm{~mm}$.

A class D power amplifier has been used for the primary network. Experimental results have shown that a power of tens of milliwatts can be transferred to a load of $100 \Omega$ placed at the secondary network up to a distance of $2 \mathrm{~cm}$, near seven times the radius of the coils $(3 \mathrm{~mm})$. The addition of a rectifier and a voltage regulator in order to properly power an autonomous sensor (3V@30 mA) limits the powering range to $1 \mathrm{~cm}$. Overall efficiencies around $45 \%$ and $20 \%$ have been achieved at distances of $5 \mathrm{~mm}$ and $1 \mathrm{~cm}$, respectively.

\section{ACKNOWLEDGMENT}

The authors acknowledge the technical support of Francis López and the car company SEAT for the initial discussions about the application.

\section{REFERENCES}

[1] C.S. Wang, O. H. Stielau, and G. A. Covic, "Design considerations for a contactless electric vehicle battery charger," IEEE Trans. Industrial Electronics, vol. 52, no. 5, pp. 1308-1314, Oct. 2005.

[2] D. C. J. Krop, E. A. Lomonova, J. W. Jansen, and J. J. H. Paulides, “A study on the integration of contactless energy transfer in the end teeth of a PM synchronous linear motor," J. Applied Physics, vol. 105, 07F115, 2009.

[3] D.M. Dobkin, The RF in RFID. Passive UFH RFID in Practice. Amsterdam: Newnes-Elsevier, 2008.

[4] B. Lenaerts and R. Puers, Omnidirectional Inductive Powering for Biomedical Implants. Springer, 2009.

[5] S.Y.R. Hui and W.W.C. Ho, "A new generation of universal contactless Battery Charging platform for portable Consumer Electronic equipment," IEEE Trans. Power Electronics, vol.20, no.3, pp. 620- 627, May 2005.

[6] A. Kurs, A. Karalis, R. Moffatt, J.D. Joannopoulos, P. Fisher, and M. Soljačić, "Wireless Power Transfer via Strongly Coupled Magnetic Resonances," Science, Vol. 317, pp. 83-866, July 2007.

[7] C. Chih-Jung, C. Tah-Hsiung, L. Chih-Lung, and J. Zeui-Chown, "A Study of Loosely Coupled Coils for Wireless Power Transfer," IEEE Trans. on Circuits and Systems II: Express Briefs, vol.57, no.7, pp.536540, July 2010.

[8] A, Kurs, R. Moffatt, and M. Soljačić, "Simultaneous mid-range power transfer to multiple devices," Appl. Phys. Lett. Vol. 96, 044102, 2010.

[9] A. K. RamRakhyani, S. Mirabbasi, and M. Chiao, "Design and Optimization of Resonance-Based Efficient Wireless Power Delivery Systems for Biomedical Implants," IEEE Trans. on Biomedical Circuits and Systems, vol.5, no.1, pp.48-63, Feb. 2011.

[10] F. Segura-Quijano, J. Garcia-Canton, J. Sacristan, T. Oses, and A. Baldi, "Wireless powering of single-chip systems with integrated coil and external wire-loop resonator," Appl. Phys. Lett., vol. 92, 074102, 2008.

[11] J. Albesa, M. Gasulla, "Seat Occupancy and Belt Detection in Removable Vehicle Seats via Inductive Coupling”, IEEE VTC2011-Fall (submitted).

[12] M.W. Baker, R. Sarpeshkar, "Feedback Analysis and Design of RF Power Links for Low-Power Bionic Systems," IEEE Trans. Biomedical Circuits and Systems, vol.1, no.1, pp.28-38, March 2007.

[13] International Commission on Non-Ionizing Radiation Protection, [ICNIRP] "Guidelines for limiting exposure to time-varying electric, magnetic, and electromagnetic fields," Health Physics, vol. 74, no. 4, April 1998.

[14] N. Mohan, T.M. Undeland, W.P. Robbins. Power Electronics. Converters, Applicatins, and Design. John Wiley \& Sons, $3^{\text {rd }}$ ed. 2003. 